\title{
Long-Time Performance of Unsplit PMLs with Explicit Second Order Schemes
}

\author{
S. Abarbanel · H. Qasimov • S. Tsynkov
}

Received: 10 May 2008 / Revised: 17 February 2009 / Accepted: 19 February 2009 /

Published online: 3 March 2009

(C) The Author(s) 2009. This article is published with open access at Springerlink.com

\begin{abstract}
A gradual long-time growth of the solution in perfectly matched layers (PMLs) has been previously reported in the literature. This undesirable phenomenon may hamper the performance of the layer, which is designed to truncate the computational domain for unsteady wave propagation problems. For unsplit PMLs, prior studies have attributed the growth to the presence of multiple eigenvalues in the amplification matrix of the governing system of differential equations. In the current paper, we analyze the temporal behavior of unsplit PMLs for some commonly used second order explicit finite-difference schemes that approximate the Maxwell's equations. Our conclusion is that on top of having the PML as a potential source of long-time growth, the type of the layer and the choice of the scheme play a major role in how rapidly this growth may manifest itself and whether or not it manifests itself at all.
\end{abstract}

Keywords Perfectly matched layer (PML) · Long-time growth · Wave propagation · Maxwell's equations · Numerical solution · Second order approximation · Yee scheme · Leap-frog scheme · Lax-Wendroff scheme · Central difference/Runge-Kutta scheme · Yee/Runge-Kutta scheme

Research supported by the US Air Force, grant number FA9550-07-1-0170, and US NSF, grant number DMS-0509695.

S. Abarbanel

School of Mathematical Sciences, Tel Aviv University, Ramat Aviv, Tel Aviv 69978, Israel

e-mail: saula@post.tau.ac.il

H. Qasimov

Max Plank Institute, Leipzig, Germany

e-mail: qasimov@mis.mpg.de

S. Tsynkov (凶)

Department of Mathematics, North Carolina State University, Box 8205, Raleigh, NC 27695, USA

e-mail: tsynkov@math.ncsu.edu 


\section{Introduction}

Perfectly matched layers (PMLs) were first introduced by Bérenger [1, 2]. They provide an efficient mechanism for truncating the computational domain when solving the problems of propagation of electromagnetic wave over unbounded regions. Beŕenger proposed to surround the computational domain by a layer of artificial material capable of rapidly attenuating all the outgoing waves while generating no reflections from the interface between the domain and the layer, regardless of the waves' frequency or angle of incidence. These capabilities were attained by splitting the field components, i.e., by introducing the additional unknowns and equations in the layer, and then using the extra degrees of freedom for the development of an efficient waves' attenuation strategy.

From the standpoint of applications, the split field PML of [1, 2] has demonstrated an overall excellent performance. It, however, has been noticed [3] (see also [4]) that the Bérenger's split, as well as other splits available in the literature [5], transform the strongly hyperbolic (symmetric) Maxwell's equations into a weakly hyperbolic system. This, in turn, implies transition from strong well-posedness to weak well-posedness of the Cauchy problem [6]. The resulting concerns about well-posedness and stability of the Bérenger's PML have prompted the development of other PMLs [7-9]. These alternative strategies do not require splitting the field components, although they still introduce some additional unknowns inside the layer. Later, however, the unsplit PMLs have also been found susceptible to gradually developing instabilities [10]. A comparative study of some of the various PMLs was performed in [11].

Two unsplit PMLs were investigated in [10] for the 2D Cartesian transverse electric (TE) Maxwell's equations. For the layer that truncates the domain in the $x$-direction, the so-called mathematically derived PML of [9] is given by:

$$
\begin{aligned}
\frac{\partial E_{x}}{\partial t} & =\frac{\partial H_{z}}{\partial y} \\
\frac{\partial E_{y}}{\partial t} & =-\frac{\partial H_{z}}{\partial x}-2 \sigma E_{y}-\sigma P \\
\frac{\partial H_{z}}{\partial t} & =\frac{\partial E_{x}}{\partial y}-\frac{\partial E_{y}}{\partial x}+\sigma^{\prime} Q \\
\frac{\partial P}{\partial t} & =\sigma E_{y} \\
\frac{\partial Q}{\partial t} & =-\sigma Q-E_{y}
\end{aligned}
$$

The additional quantities $P$ and $Q$ are non-zero only in the layer, and the damping of waves is controlled by the parameter $\sigma(x)=\frac{(x-a)^{3}}{d^{3}}$, where $d$ is the PML thickness and $x=a$ is the interface between the domain and the layer.

An alternative to (1) is the physically motivated unsplit PML of [7] or [8], which can be written in the following form [9]:

$$
\begin{aligned}
\frac{\partial E_{x}}{\partial t} & =\frac{\partial H_{z}}{\partial y}+\sigma\left(E_{x}-P\right) \\
\frac{\partial E_{y}}{\partial t} & =-\frac{\partial H_{z}}{\partial x}-\sigma E_{y}
\end{aligned}
$$




$$
\begin{aligned}
\frac{\partial H_{z}}{\partial t} & =\frac{\partial E_{x}}{\partial y}-\frac{\partial E_{y}}{\partial x}-\sigma H_{z} \\
\frac{\partial P}{\partial t} & =\sigma\left(E_{x}-P\right)
\end{aligned}
$$

Unlike in (1), there is only one additional quantity, $P$, inside the PML (2).

The analysis of [10] focuses on the quiescent solutions inside the PML, long after the initial perturbation has been absorbed. It is shown that if the spatial derivatives are neglected, then the system of ODEs that results from either (1) or (2) would be characterized by multiple eigenvalues and the degeneracy of eigenvectors. This indicates the possibility of a polynomial growth. Experimentally, a slow growth originating in the PML was indeed observed in [10] for one specific choice of the discrete approximation, namely when (1) or (2) were approximated using fourth order central differences in space accompanied by a fourth order Runge-Kutta method in time.

On the other hand, there have been reports in the literature that for a given computational setup the growth may or may not be observable, and that the artifacts generated in the PML, if any, may or may not propagate back into the computational domain [12]. Hence, in the current paper we set our goal as to systematically study the long-time behavior of the PMLs (1) and (2) with a number of commonly used explicit second order finite-difference schemes. Specifically, we consider two versions of the popular Yee scheme-the original fully staggered Yee scheme of [13] and the scheme obtained by second order staggered Yee differences in space combined with the Runge-Kutta evolution in time. We also consider the leap-frog scheme, the Lax-Wendroff scheme, and the central difference scheme supplemented by the Runge-Kutta time integration (subsequently referred to as the central/RK scheme). Compared to [10], we complement our analysis by looking at the eigenvalues (amplification factors) of the actual discretizations, as opposed to only those of the differential problem. Besides, we conduct extensive numerical tests for each of the aforementioned schemes.

It turns out that the growth can, in fact, be effectively removed by just the discretization itself. The Yee scheme, the Lax-Wendroff scheme, and the central/RK scheme applied to the PML (2) exhibit no growth at all in our experiments, whereas both Yee-type schemes, as well as the Lax-Wendroff scheme, applied to the PML (1) exhibit a very slow growth that can be deemed non-existent for any practical purpose. On the other hand, the discretization may also greatly exacerbate the growth and make the computations impossible, which is what happens to the leap-frog scheme with both PMLs. Finally, the popular family of central schemes with Runge-Kutta smoothers ${ }^{1}$ remains susceptible to a moderately slow growth of the solution in the PML (1), which indicates that the appropriate remedies need to be sought for. Altogether, we conclude that the phenomenon of growth of the numerical solution in the PML proves to depend strongly on both the choice of the PML and the choice of the scheme.

\section{Computational Setup}

To enable an easier comparison, we take the same setup as in [10]. Our computational domain is a Cartesian square: $\{(x, y) \mid-a \leq x \leq a,-a \leq y \leq a\}$ with $a=50$; it is terminated in the $x$-direction by two symmetrically located PMLs: $-a-d \leq x \leq-a$ and $a \leq x \leq a+d$,

\footnotetext{
${ }^{1}$ It is apparently the most straightforward venue to high order approximations.
} 
where $d=10$. The Cartesian discretization grid has square cells: $h_{x}=h_{y}=1$. At the top and bottom boundaries, $y=a$ and $y=-a$, as well as at the outer boundaries of the PML, $x=a+d$ and $x=-a-d$, we use classical locally one-dimensional characteristic artificial boundary conditions (ABCs, see [14]) obtained by setting to zero the Riemann invariant that corresponds to the incoming characteristic. We emphasize that having the PML only in one coordinate direction allows us to avoid the corner problem, which may be a source of additional numerical difficulties. As such, our study focuses on the issues that can be attributed solely to the PML.

In all forthcoming simulations, we investigate numerically the evolution on the grid of a smooth initial perturbation of the magnetic field:

$$
H(x, y, 0)= \begin{cases}\cos ^{8}\left(\frac{\pi \sqrt{x^{2}+y^{2}}}{2 r_{0}}\right), & \text { if } \sqrt{x^{2}+y^{2}} \leq r_{0} \\ 0, & \text { if } \sqrt{x^{2}+y^{2}}>r_{0}\end{cases}
$$

where $r_{0}=10$. The initial values of the electric field $E_{x}$ and $E_{y}$ are zero. This is a compact initial condition, and the resulting solution is expected in the form of a wave that exits the computational domain leaving behind a vanishing "tail" (note, this is a 2D setting with no Huygens' principle). The case with a continuously operating forcing function is studied in [15], and growth of the solution similar to that reported hereafter is also observed.

We additionally emphasize that as we terminate the PML by characteristic ABCs, we can determine conclusively whether or not the long-term growth, in case it is observed, shall actually be attributed to the PML. Indeed, we can first run the computation with the PML. Then, we can merely turn off the PML (by setting $\sigma(x) \equiv 0$ ) while leaving all other components of the algorithm intact and while still keeping all the boundaries nonreflecting (to the degree permitted by the locally one-dimensional treatment). If no growth is detected in the second case (no PML), then the previously observed growth is clearly due to the PML and nothing else. Hereafter, if the growth is reported, it shall be assumed that it has been verified this way.

Finally, let us quantify the notion of a long time. Introduce the unit of time as the domain size over the propagation speed: $T=2 a / c$, where $c=1$. We will be investigating the phenomena that start manifesting themselves no earlier than $10 T$ to $15 T$ and all the way up to hundreds and thousands of $T$ 's.

\section{The Original Yee Scheme}

The staggered Yee scheme [13] as applied to the mathematical PML (1) reads:

$$
\begin{aligned}
\frac{E_{x_{m, n+1 / 2}^{p+1 / 2}}^{p}-E_{x_{m, n+1 / 2}^{p-1 / 2}}=}{\tau} & \frac{H_{m, n+1}^{p}-H_{m, n}^{p}}{h_{y}} \\
\frac{E_{y_{m+1 / 2, n}^{p+1 / 2}}^{p}-E_{y_{m+1 / 2, n}^{p-1 / 2}}^{p}=}{\tau} & -\frac{H_{m+1, n}^{p}-H_{m, n}^{p}}{h_{x}} \\
& -2 \sigma_{m+1 / 2} \frac{E_{y_{m+1 / 2, n}^{p+1 / 2}}^{p}+E_{y_{m+1 / 2, n}^{p-1 / 2}}^{p}}{2}-\sigma_{m+1 / 2} P_{m+1 / 2, n}^{p}
\end{aligned}
$$

$$
\frac{P_{m+1 / 2, n}^{p+1}-P_{m+1 / 2, n}^{p}}{\tau}=\sigma_{m+1 / 2} E_{y_{m+1 / 2, n}^{p+1 / 2}}^{p}
$$




$$
\begin{aligned}
\frac{Q_{m, n}^{p+1}-Q_{m, n}^{p}}{\tau}= & -\sigma_{m} \frac{Q_{m, n}^{p+1}+Q_{m, n}^{p}}{2}-\frac{E_{y_{m+1 / 2, n}}^{p+1 / 2}+E_{y_{m-1 / 2, n}}^{p+1 / 2}}{2} \\
\frac{H_{m, n}^{p+1}-H_{m, n}^{p}}{\tau}= & \frac{E_{x_{m, n+1 / 2}^{p+1 / 2}}^{p+E_{x, n-1 / 2}^{p+1 / 2}}}{h_{y}} \\
& -\frac{E_{y_{m+1 / 2, n}}^{p+1 / 2} E_{y_{m-1 / 2, n}^{p+1 / 2}}^{p}}{h_{x}}+\sigma_{m}^{\prime} \frac{Q_{m, n}^{p+1}+Q_{m, n}^{p}}{2}
\end{aligned}
$$

where $H_{z} \equiv H$, the indices $m, n$, and $p$ correspond to $x, y$, and $t$, respectively, and the equations for $E_{y}$ and $Q$ are approximated semi-implicitly. For the PML (2), the approximation using Yee scheme looks similar. In actual numerical runs, the Yee scheme exhibits no growth with the physical PML (2). More specifically, the magnitude of the solution decays gradually and does not exceed $10^{-6}$ at $t=2500 T$. From the standpoint of practice, this value is extremely large, and hence we can say that the growth does not manifest itself at all. For the mathematical PML (1), scheme (3) still displays some growth but it is very slow; no artifacts can be seen until several hundred $T$.

In Fig. 1, we are showing a typical snapshot of the solution obtained using scheme (3)the level lines of $E_{x}(x, y, t)$ at $t=500 T$. Other quantities look similar, their magnitude across the domain does not exceed 0.5. In practice, the time $t=500 T$ is also very large and we therefore believe that the growth shown in Fig. 1 can be disregarded in most cases. Moreover, one can see from Fig. 1 that the artifacts originate in the PML near its interface with the domain, i.e., in the region where $\sigma(x)$ is small. This observation is generally in agreement with the analysis of [10].

It is also interesting to see what happens to the eigenvalues. We adopt an approach similar to that of [10] and analyze quiescent solutions in the PML, i.e., assume that there is no spatial variation and neglect all spatial differences. Then, the difference equations for $E_{y}$ and $P$ decouple from the rest of system (3), and dropping $m$ and $n$ for convenience, we have (at a given location):

$$
\begin{aligned}
\frac{E_{y}{ }^{p+1 / 2}-E_{y}{ }^{p-1 / 2}}{\tau} & =-\sigma\left(E_{y}{ }^{p+1 / 2}+E_{y}{ }^{p-1 / 2}\right)-\sigma P^{p} \\
\frac{P^{p+1}-P^{p}}{\tau} & =\sigma E_{y}{ }^{p+1 / 2}
\end{aligned}
$$

Fig. $1 E_{x}$ for the Yee scheme (3) at $t=500 T$; the contours are equally spaced between -0.475 and 0.475 with the increment 0.05

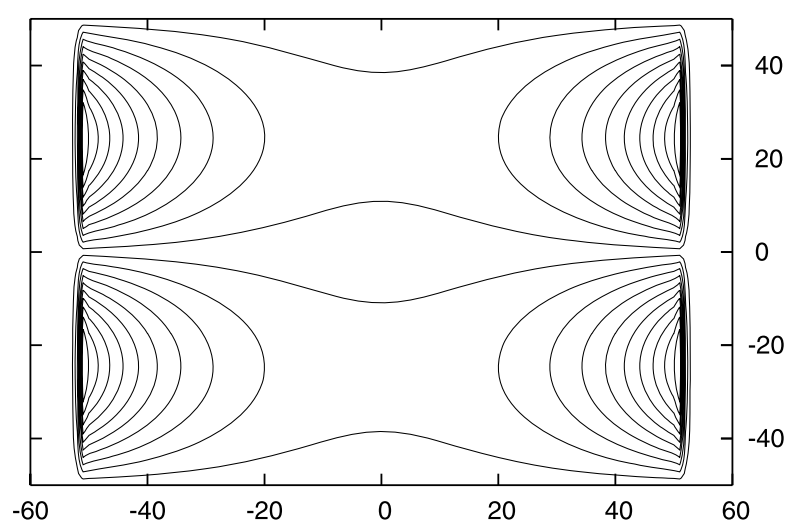


System (4) is a second order system of ordinary difference equations. Its eigenvalues can be easily computed: $\lambda_{1}=1 /(1+\sigma \tau)$ and $\lambda_{2}=1-\sigma \tau$. Compared to the continuous case [10], we observe a split of the eigenvalue, because the differential counterpart of system (4) has a degenerate eigenvalue $\lambda=-\sigma$. The differential physical PML (2) in the quiescent state is characterized by a double eigenvalue $\lambda=0$ with only one eigenvector. For the Yee scheme, however, it translates into a degenerate double eigenvalue as well, $\lambda=1$, i.e., there is no split. We note that in [10], the growth of the solution in the PML (1) was attributed precisely to the degenerate eigenvalue $\lambda=-\sigma$. Yet our numerical experiments with the Yee scheme show that this growth, no matter how slow it is, is present for the PML (1), for which the double eigenvalue gets split, and is not present for the PML (2), for which it stays unsplit.

\section{The Leap-Frog Scheme}

Let us write down the leap-frog scheme as it applies to system (2):

$$
\begin{aligned}
\frac{E_{x_{m, n}}^{p+1}-E_{x_{m, n}}^{p-1}}{2 \tau} & =\frac{H_{m, n+1}^{p}-H_{m, n-1}^{p}}{2 h_{y}}+\sigma_{m}\left(E_{x_{m, n}}^{p}-P_{m, n}^{p}\right) \\
\frac{E_{y_{m, n}^{p+1}}^{p+1}-E_{y_{m, n}^{p-1}}^{p-1}}{2 \tau} & =-\frac{H_{m+1, n}^{p}-H_{m-1, n}^{p}}{2 h_{x}}-\sigma_{m} E_{y_{m, n}^{p}}^{p} \\
\frac{H_{m, n}^{p+1}-H_{m, n}^{p-1}}{2 \tau} & =\frac{E_{x_{m, n+1}}^{p}-E_{x_{m, n-1}}^{p}}{2 h_{y}}-\frac{E_{y_{m+1, n}}^{p}-E_{y_{m-1, n}}^{p}}{2 h_{x}}-\sigma_{m} H_{m, n}^{p} \\
\frac{P_{m, n}^{p+1}-P_{m, n}^{p-1}}{2 \tau} & =\sigma_{m}\left(E_{x_{m, n}}^{p}-P_{m, n}^{p}\right)
\end{aligned}
$$

We will use the principle of frozen coefficients and investigate the von Neumann stability of scheme (5) for a fixed value of $\sigma \neq 0$. Substituting the solution $q \lambda^{p} e^{i(\alpha m+\beta n)}$ into (5), where $\boldsymbol{q}$ is a 4-dimensional vector and $\alpha$ and $\beta$ are the frequencies that correspond to $x$ and $y$, we require that the corresponding determinant be zero and obtain $\left(h_{x}=h_{y}=h\right)$ :

$$
4 \lambda^{2}\left(\lambda^{2}-1\right)^{2} \tau^{2} \sin ^{2} \alpha+\left(-1+\lambda^{2}+2 \lambda \sigma \tau\right)^{2}\left(h^{2}\left(\lambda^{2}-1\right)^{2}+4 \lambda^{2} \tau^{2} \sin ^{2} \beta\right)=0
$$

An algebraic equation of degree 8 is impossible to solve in the general case. However, for the simplest case of a quiescent solution, $\alpha=\beta=0$, we have:

$$
\lambda_{1,2}=-\sigma \tau-\sqrt{1+\sigma^{2} \tau^{2}}, \quad \lambda_{3,4}=-\sigma \tau+\sqrt{1+\sigma^{2} \tau^{2}}, \quad \text { and } \quad \lambda_{5,6,7,8}=1
$$

We see that the eigenvalues $\lambda_{1,2}$ are located outside the unit disk. Still, for sufficiently small time steps we can always claim that $\left|\lambda_{1,2}\right| \leq 1+$ const $\tau$. Hence, the situation is not "fatal," and scheme (5) may remain formally stable for finite time intervals, ${ }^{2}$ or, more precisely, the necessary von Neumann condition of stability may still hold, provided that the other eigenvalues that correspond to non-zero frequencies will not "misbehave." However, the stability constant becomes exponentially large and obviously cannot grow slower than an exponential function of time even if all $\alpha \neq 0$ and $\beta \neq 0$ are taken into account. In practice, the computation is completely ruined already at short times $t \sim T$. The results for the mathematical PML (1) are virtually identical.

\footnotetext{
${ }^{2}$ For long time stability, the eigenvalues have to be strictly inside the unit circle.
} 


\section{The Lax-Wendroff Scheme}

The Lax-Wendroff scheme for either type of the PML is obtained in the usual way, by reexpressing the second time derivative of each unknown quantity through the corresponding equation(s) and subsequently canceling the $\mathcal{O}(\tau)$ term in the truncation error by subtraction. For PML (1), this yields:

$$
\begin{aligned}
& \frac{E_{x m, n}^{p+1}-E_{x m, n}^{p}}{\tau}=\frac{H_{m, n+1}^{p}-H_{m, n-1}^{p}}{2 h_{y}}+\frac{\tau}{2}[\underbrace{\frac{\partial^{2} E_{x}}{\partial y^{2}}-\frac{\partial^{2} E_{y}}{\partial x \partial y}+\sigma^{\prime} \frac{\partial Q}{\partial y}}_{\mathcal{O}\left(h^{2}\right) \text { by central differences }}]_{m, n}^{p} \\
& \frac{E_{y_{m, n}^{p+1}}^{p+E_{y_{m, n}}^{p}}}{\tau}=-\frac{H_{m+1, n}^{p}-H_{m-1, n}^{p}}{2 h_{x}}-2 \sigma_{m} E_{y_{m, n}}^{p}-\sigma_{m} P_{m, n}^{p} \\
& +\frac{\tau}{2}[\underbrace{\frac{\partial^{2} E_{y}}{\partial x^{2}}-\frac{\partial^{2} E_{x}}{\partial y \partial x}-\sigma^{\prime} \frac{\partial Q}{\partial x}}_{\mathcal{O}\left(h^{2}\right) \text { by central differences }}-\sigma^{\prime \prime} Q-\underbrace{2 \sigma \frac{\partial E_{y}}{\partial t}-\sigma \frac{\partial P}{\partial t}}_{\begin{array}{c}
\text { via the equations } \\
\text { then also } \mathcal{O}\left(h^{2}\right)
\end{array}}]_{m, n}^{p} \\
& \frac{H_{m, n}^{p+1}-H_{m, n}^{p}}{\tau}=\frac{E_{x m, n+1}^{p}-E_{x_{m, n-1}}^{p}}{2 h_{y}}-\frac{E_{y_{m+1, n}^{p}}^{p}-E_{y_{m-1, n}^{p}}^{p}}{2 h_{x}}+\sigma_{m}^{\prime} Q_{m, n}^{p} \\
& +\frac{\tau}{2}[\underbrace{\frac{\partial^{2} H}{\partial x^{2}}-\frac{\partial^{2} H}{\partial y^{2}}+2 \sigma \frac{\partial E_{y}}{\partial x}+\sigma \frac{\partial P}{\partial x}}_{\mathcal{O}\left(h^{2}\right) \text { by central differences }} \\
& \left.+2 \sigma^{\prime} E_{y}+\sigma^{\prime} P-\sigma^{\prime}\left(\sigma Q+E_{y}\right)\right]_{m, n}^{p} \\
& \frac{P_{m, n}^{p+1}-P_{m, n}^{p}}{\tau}=\sigma_{m} E_{y_{m, n}}^{p}+\frac{\tau}{2} \sigma\left[-\frac{\partial H}{\partial x}-2 \sigma E_{y}-\sigma P\right]_{m, n}^{p} \\
& \frac{Q_{m, n}^{p+1}-Q_{m, n}^{p}}{\tau}=-\sigma_{m} Q_{m, n}^{p}-E_{y_{m, n}}^{p}+\frac{\tau}{2}\left[\sigma^{2} Q+3 \sigma E_{y}+\sigma P+\frac{\partial H}{\partial x}\right]_{m, n}^{p}
\end{aligned}
$$

In the quiescent state, equations for $E_{y}$ and $P$ decouple from system (6), cf. (4). Then, analysis of the eigenvalues similar to the one conducted in Sect. 3 yields: $\lambda_{1,2}=1-\sigma \tau+$ $\frac{\sigma^{2} \tau^{2}}{2}$. We see that the multiple eigenvalue from the differential system does not get split by the discretization.

There is, however, an alternative way of discretizing the ODEs from system (1). Namely, one can use a semi-implicit (Crank-Nicolson type) scheme for these two equations and still maintain the overall second order accuracy. In this case, the continuous multiple eigenvalue gets split by the discretization:

$$
\lambda_{1,2}=1-\sigma \tau+\frac{\sigma^{2} \tau^{2}}{2}+\frac{\sigma^{3} \tau^{3}}{4} \pm \frac{\sigma^{2} \tau^{2}}{4} \sqrt{-4+4 \sigma \tau+\sigma^{2} \tau^{2}}
$$

Yet in our numerical experiments no difference in the long-time behavior has been observed between scheme (6) and the Lax-Wendroff scheme with the ODEs approximated semi-implicitly. Both schemes develop a very slow growth, with the artifacts becoming noticeable at about 500T, see Fig. 2. In accordance with the observations of [10], they originate near the interface between the domain and the layer. 
Fig. $2 E_{x}$ for the Law-Wendroff scheme (6) at $t=500 T$; the contours are equally spaced between -0.575 and 0.575 with the increment 0.05

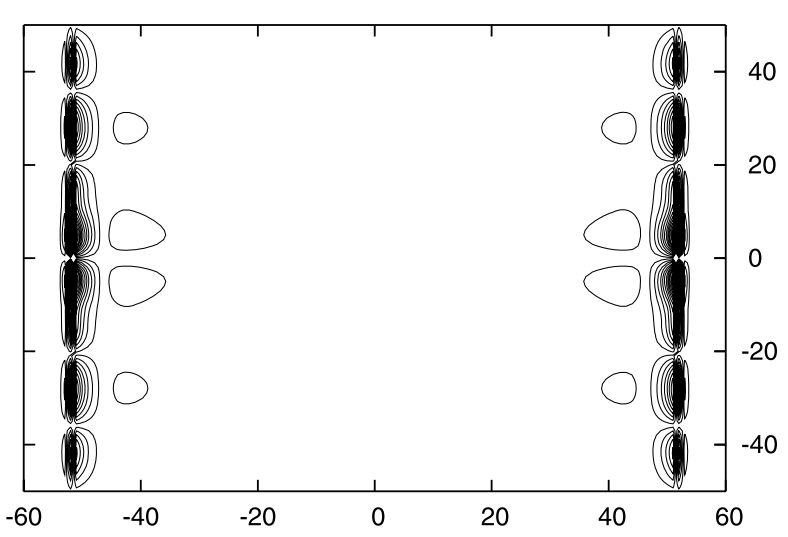

The Lax-Wendroff scheme for the physical PML (2) can be built in much the same way as scheme (6). In the experiments, PML (2) exhibits no artificial growth at least until $t=$ $2500 T$. Altogether, this behavior is very similar to what we have seen for the Yee scheme in Sect. 3.

It should also be noted that the implementation of scheme (6) requires one special consideration. Originally, it was noticed that the artifacts in the solution could develop much more rapidly than shown in Fig. 2, and starting predominantly at the intersections of the interfaces between the domain and the PML $x= \pm a$ with the lateral boundaries $y= \pm a$. This was attributed to the presence of the term $\sigma^{\prime \prime} Q$ in the equation for $E_{y}$ in system (6). For the cubic profile of $\sigma(x)$, this term has relatively low regularity at the interfaces $x= \pm a$, it is continuous with discontinuous first derivative. If, however, this term is merely removed, the growth displayed by scheme (6) slows down very considerably and does not noticeably manifest itself until several hundred $T$. In doing so, inside the computational domain the scheme still remains a complete second order Lax-Wendroff.

\section{The Central Difference/RK Scheme}

Let $\boldsymbol{q}_{m, n}^{p}$ denote the complete vector of unknowns at the spatial location $(m, n)$ and time level $p$. This vector has five components for the PML (1) and four components for the PML (2). Let $\boldsymbol{G}(\boldsymbol{q})$ denote the operator of spatial discretization. It replaces the $x$ and $y$ partial derivatives on the right-hand side of either (1) or (2) by the corresponding second order central differences. It also replaces the non-differentiated terms by the appropriate nodal values. Then, the central difference second order scheme for either system (1) or system (2) with the standard fourth order Runge-Kutta evolution in time is given by:

$$
\frac{\boldsymbol{q}_{m, n}^{p+1}-\boldsymbol{q}_{m, n}^{p}}{\tau}=\frac{1}{6}\left(\boldsymbol{k}_{1}+2 \boldsymbol{k}_{2}+2 \boldsymbol{k}_{3}+\boldsymbol{k}_{4}\right)
$$

where

$$
\begin{array}{ll}
\boldsymbol{k}_{1}=\boldsymbol{G}\left(\boldsymbol{q}_{m, n}^{p}\right), & \boldsymbol{k}_{2}=\boldsymbol{G}\left(\boldsymbol{q}_{m, n}^{p}+\tau \boldsymbol{k}_{1} / 2\right) \\
\boldsymbol{k}_{3}=\boldsymbol{G}\left(\boldsymbol{q}_{m, n}^{p}+\tau \boldsymbol{k}_{2} / 2\right), & \boldsymbol{k}_{4}=\boldsymbol{G}\left(\boldsymbol{q}_{m, n}^{p}+\tau \boldsymbol{k}_{3}\right)
\end{array}
$$


Fig. $3 H$ for the central/RK scheme (7a)-(7b) applied to the PML (1) at $t=15 T$; the contours are equally spaced between -0.4 and 0.4 with the increment 0.1 and with the exception of the zero contour

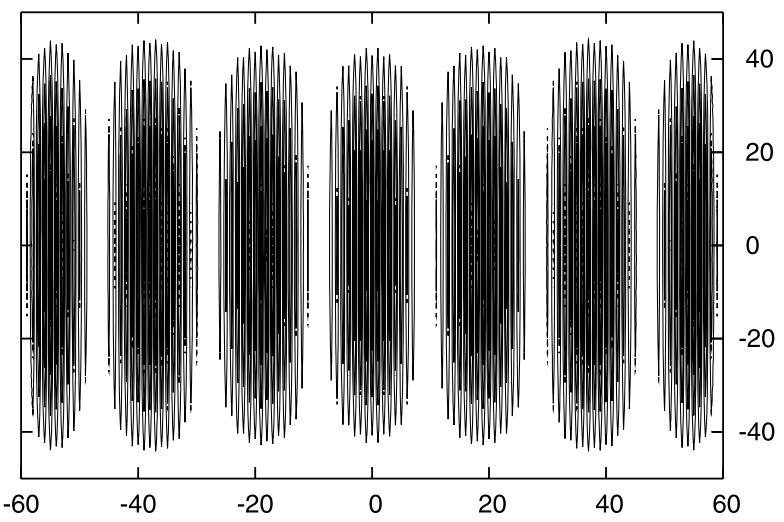

Alternatively, one can use a third order Runge-Kutta evolution in time, which yields:

$$
\frac{\boldsymbol{q}_{m, n}^{p+1}-\boldsymbol{q}_{m, n}^{p}}{\tau}=\frac{1}{4}\left(\boldsymbol{k}_{1}+3 \boldsymbol{k}_{3}\right)
$$

where

$$
\boldsymbol{k}_{1}=\boldsymbol{G}\left(\boldsymbol{q}_{m, n}^{p}\right), \quad \boldsymbol{k}_{2}=\boldsymbol{G}\left(\boldsymbol{q}_{m, n}^{p}+\tau \boldsymbol{k}_{1} / 3\right), \quad \boldsymbol{k}_{3}=\boldsymbol{G}\left(\boldsymbol{q}_{m, n}^{p}+2 \tau \boldsymbol{k}_{2} / 3\right)
$$

Note that second order Runge-Kutta methods cannot be applied to the central difference spatial discretization $\boldsymbol{G}(\boldsymbol{q})$ because the resulting scheme will be unstable. In doing so, the instability will not be due to the PML, it will rather be a genuine von Neumann instability. On the other hand, both schemes $(7 a)-(7 b)$ and $(8 a)-(8 b)$ are stable, provided that the Courant number does not exceed 2.8 for scheme (7a)-(7b) and about 1.7 for scheme (8a)-(8b).

Analysis of the eigenvalues in the quiescent state (similar to the analysis conducted in Sects. 3 and 5) shows that either scheme, (7a)-(7b) or (8a)-(8b), applied to either system, (1) or (2), preserves multiple eigenvalues that characterize the differential formulation.

As far as the numerical results, both schemes (7a)-(7b) and (8a)-(8b) applied to the mathematical PML (1) produce a moderate growth, with the artifacts clearly observable already for the times $t$ ranging between $10 T$ and $15 T$, see Fig. 3. Furthermore, unlike in the previous settings (Sects. 3 and 5), we could not see here the artifacts developing in the layer and then propagating toward the domain. We rather saw them gradually picking up throughout the entire computational region, as Fig. 3 shows. We are completely sure though that these artifacts are still due to the layer and nothing else, because it has been verified by switching off the PML while keeping all other parameters intact, as explained in Sect. 2. Moreover, it has also been verified that these artifacts are not due to any von Neumann type instability. They develop with the same rate (with respect to the actual time rather than the number of steps) for the Courant number taken on the borderline of stability region, as well as for the three times smaller Courant number.

For the physical PML (2), no growth of the Runge-Kutta solution could be observed at least until $t=2500 T$. This is in contrast with the findings of [10], although in [10] the differences in space were fourth order, and the code was multi-block with characteristicsbased treatment of the interfaces. 
Fig. $4 E_{x}$ for the Yee/RK scheme applied to the PML (1) at $t=500 T$; the contours are equally spaced between -0.475 and 0.475 with the increment 0.05

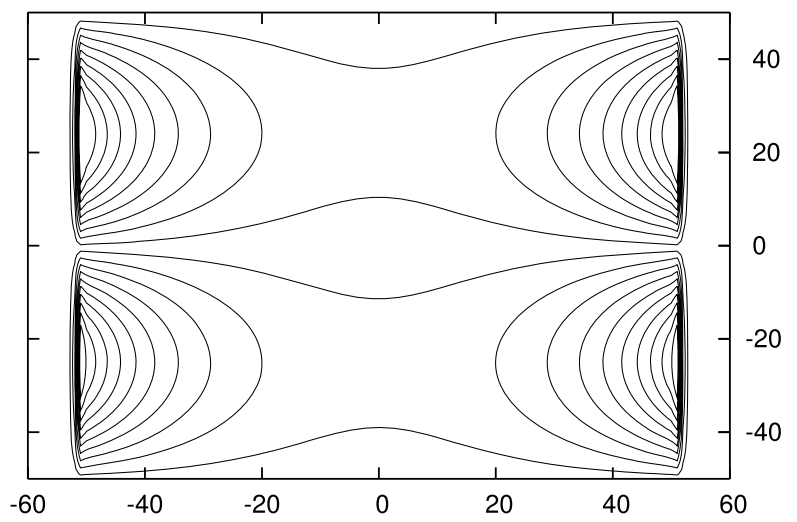

\section{The Yee/RK Scheme}

In Sects. 3 and 6, we have seen that the behavior of the original Yee scheme and that of the central/RK scheme differ noticeably from one another. Hence, we decided to include a combination scheme in our study that has Yee-type staggered differences in space, see formula (3), and four stage Runge-Kutta evolution in time, see formulae (7a)-(7b). In doing so, there is no staggering of the variables in time. The resulting Yee/RK scheme is a also a popular tool among the CEM practitioners.

Analysis of the quiescent state eigenvalues for this scheme is exactly the same as the corresponding analysis for the central/RK scheme, which we conducted in Sect. 6. Indeed, once all the spatial differences are dropped, the two schemes reduce to one another. The analysis shows that multiple eigenvalues that characterize the continuous formulation of the problem are also preserved by the discretization.

Computational behavior of the combined Yee/RK scheme appears very similar to that of the original Yee scheme, see Sect. 3. In Fig. 4, we are showing the level lines for the electric field $E_{x}$ at $t=500 T$. It is easy to see that this plot closely resembles the corresponding plot for the original Yee scheme that we are showing in Fig. 1. We therefore conclude that the faster growth of the solution due to the PML that we observed in Sect. 6 for the central/RK scheme should mostly be attributed to the type of spatial discretization rather than to the type of time integration employed. This also shows that the analysis of eigenvalues in the quiescent state only may be insufficient.

\section{Summary}

We summarize our observations and results of the analysis in Table 1.

Altogether, the physical unsplit PML (2) seems somewhat less susceptible to developing the long-time growth. For the mathematical PML (1), when it is used with either type of the Yee scheme or the Lax-Wendroff scheme, the growth can still be disregarded for all practical purposes. This growth, however, is more rapid for the central difference scheme with Runge-Kutta evolution in time.

We realize, of course, that the analysis in this paper is somewhat limited in its scope. For example, we cannot say ahead of time what's going to happen to the reader's favorite high order scheme if applied to one of the PMLs we have considered. On the other hand, we have analyzed the performance of all commonly used second order explicit finite-difference 
Table 1 Long-time behavior of unsplit PMLs with second order schemes.

\begin{tabular}{|c|c|c|c|c|}
\hline & & \multicolumn{2}{|l|}{ Schemes } & \\
\hline & & Yee & $\overline{\text { Leap-frog }}$ & \\
\hline mathemat. & growth & $\sim 500 T$ & rapid & \\
\hline PML (1) & eigenvalues & split & $|\lambda|>1$ & \\
\hline physical & growth & no & rapid & \\
\hline PML (2) & eigenvalues & multiple & $|\lambda|>1$ & \\
\hline \multicolumn{5}{|l|}{ Schemes } \\
\hline \multicolumn{2}{|l|}{ Lax-Wendroff } & \multicolumn{2}{|l|}{ Central/RK } & Yee/RK \\
\hline expl. ODEs & impl. ODEs & 4-th RK & 3-rd RK & 4-th RK \\
\hline$\sim 500 T$ & $\sim 500 T$ & $\sim 15 T$ & $\sim 15 T$ & $\sim 500 T$ \\
\hline multiple & split & multiple & multiple & multiple \\
\hline no & & no & & \\
\hline multiple & & multiple & multiple & multiple \\
\hline
\end{tabular}

schemes, and within this class of methods reliable predictions can be made based on the data from Table 1. Otherwise, analysis of the eigenvalues for a discretization not covered in this paper can be performed similarly. It should be mentioned though that changing the spatial discretization alone could noticeably change the numerical behavior, as shown by the comparison of the central/RK scheme (Sect. 6) and the Yee/RK scheme (Sect. 7). Hence, the quiescent eigenvalue analysis following [10] may need to be supplemented by a more comprehensive study in the future that would enable more reliable predictions of the PML performance.

To summarize, the presence or absence of the growth of numerical solution inside the PML seems to be strongly affected by both the type of the layer and the type of discretization, in particular, the discretization in space. Whereas in many cases no practically significant growth can be observed, there are nonetheless distinct situations when the growth clearly manifests itself. In our opinion, these observations justify the development of special methodologies for stabilizing the PMLs over long propagation times. One particular methodology that achieves this goal has been proposed in our recent paper [15]; it employs the lacunae-based integration in time along with the PML. Work [15] also addresses the case of continuous excitation in time, as opposed to the solutions driven only by initial conditions that we have studied in the current paper.

Acknowledgement We would like to thank the anonymous referee for his/her helpful comments, and in particular, for the suggestion to include the Yee/RK scheme (Sect. 7) in the study.

Open Access This article is distributed under the terms of the Creative Commons Attribution Noncommercial License which permits any noncommercial use, distribution, and reproduction in any medium, provided the original author(s) and source are credited.

\section{References}

1. Bérenger, J.-P.: A perfectly matched layer for the absorption of electromagnetic waves. J. Comput. Phys. 114(2), 185-200 (1994)

2. Bérenger, J.-P.: Three-dimensional perfectly matched layer for the absorption of electromagnetic waves. J. Comput. Phys. 127(2), 363-379 (1996) 
3. Abarbanel, S., Gottlieb, D.: A mathematical analysis of the PML method. J. Comput. Phys. 134(2), 357-363 (1997)

4. Bécache, E., Joly, P.: On the analysis of Bérenger's perfectly matched layers for Maxwell's equations. Model. Math. Numer. Anal. 36(1), 87-119 (2002)

5. Cummer, S.A.: A simple, nearly perfectly matched layer for general electromagnetic media. IEEE Microw. Wirel. Compon. Lett. 13(3), 128-130 (2003)

6. Kreiss, H.-O., Lorenz, J.: Initial-Boundary Value Problems and the Navier-Stokes Equations. Pure and Applied Mathematics, vol. 136. Academic Press, San Diego (1989)

7. Gedney, S.D.: An anisotropic perfectly matched layer-absorbing medium for the truncation of FDTD lattices. IEEE Trans. Antennas Propag. 44(12), 1630-1639 (1996)

8. Ziolkowski, R.W.: Time-derivative Lorenz material model based absorbing boundary conditions. IEEE Trans. Antennas Propag. 45(10), 1530-1535 (1997)

9. Abarbanel, S., Gottlieb, D.: On the construction and analysis of absorbing layers in CEM. Appl. Numer. Math. 27(4), 331-340 (1998)

10. Abarbanel, S., Gottlieb, D., Hesthaven, J.S.: Long time behavior of the perfectly matched layer equations in computational electromagnetics. J. Sci. Comput. 17(14), 405-422 (2002)

11. Turkel, E., Yefet, A.: Absorbing PML boundary layers for wave-like equations. Appl. Numer. Math. 27, 533-557 (1998)

12. Bécache, E., Petropoulos, P.G., Gedney, S.D.: On the long-time behavior of unsplit perfectly matched layers. IEEE Trans. Antennas Propag. 52(5), 1335-1342 (2004)

13. Yee, K.S.: Numerical solution of initial boundary value problem involving Maxwell's equations in isotropic media. IEEE Trans. Antennas Propag. 14, 302-307 (1966)

14. Tsynkov, S.V.: Numerical solution of problems on unbounded domains. A review. Appl. Numer. Math. 27, 465-532 (1998)

15. Qasimov, H., Tsynkov, S.: Lacunae based stabilization of PMLs. J. Comput. Phys. 227, 7322-7345 (2008) 